\title{
FATOR DE SENSIBILIDADE AO DÉFICIT HÍDRICO DA CULTURA DO FEIJÃO CAUPI (VIGNA UNGUICULATA (L.) WALP.) ${ }^{1}$
}

\author{
Lucileudo Gomes Cordeiro², Francisco Marcus Lima Bezerra ${ }^{3}$, \\ João José Anselmo dos Santos ${ }^{2}$ e Eugênio Paceli de Miranda ${ }^{2}$
}

\begin{abstract}
RESUMO
Este trabalho foi conduzido na área experimental do Laboratório de Hidráulica e Irrigação da Universidade Federal do Ceará, Fortaleza, CE, com o objetivo de determinar o fator de rendimento da cultura (ky) do feijão caupi, sob regime de déficit hídrico em seus estádios fenológicos. O delineamento experimental adotado foi o de blocos ao acaso, com quatro repetições e oito tratamentos, os quais foram definidos em função da época de indução do déficit hídrico nos diferentes estádios fenológicos do feijão, correspondendo à ocorrência de déficit hídrico em um, dois ou três estádios; este déficit foi obtido aplicando-se metade da lâmina usada para o tratamento sem déficit. As evapotranspirações máxima e real da cultura do feijão foram determinadas pelo método do balanço hídrico e os coeficientes de sensibilidade $\left(\mathrm{k}_{\mathrm{y}}\right)$ do feijão ao déficit hídrico o foram através da expressão $\mathrm{k}_{\mathrm{y}}=\left[1-\left(\mathrm{y}_{\mathrm{r}} / \mathrm{y}_{\mathrm{m}}\right)\right] /\left[1-\left(\mathrm{ET}_{\mathrm{r}} / \mathrm{ET}_{\mathrm{m}}\right)\right]$. O estádio de enchimento de grãos foi o mais sensível ao déficit hídrico quando este foi aplicado em apenas um estádio; os valores do $\left(\mathrm{k}_{\mathrm{y}}\right)$ foram inferiores a 1 , indicando baixa sensibilidade da cultura ao estresse hídrico.
\end{abstract}

Palavras-chave: Vigna ungüiculata, déficit hídrico, evapotranspiração

\section{THE COW PEA BEAN (VIGNA UNGÜICULATA (L.) WALP.) SENSIBILITY FACTOR TO THE WATER DEFICIT}

\begin{abstract}
The study was conducted in the Hydraulic Irrigation Laboratory Experimental Area of the Federal University of Ceará in order to determine the cow pea bean crop yield factor $\left(\mathrm{k}_{\mathrm{y}}\right)$ under water deficit at different phenological stages. The experimental design consisted of entirely randomized blocks with eight treatments and four replicates. The treatments were established according to the time of the water deficit induction at the crop phenological stages and that corresponded to the occurence of the water deficit at one, two or three stages. The water deficit was obtained by applying half of the water depth used for the non-deficit treatment. The maximum and real cow pea crop evapotranspirations were determined by using the water balance method and the crop water deficit sensibility coefficients were calculated by using the equation $\mathrm{k}_{\mathrm{y}}=\left[1-\left(\mathrm{y}_{\mathrm{r}} / \mathrm{y}_{\mathrm{m}}\right)\right] /\left[1-\left(\mathrm{ET}_{\mathrm{r}} / \mathrm{ET}_{\mathrm{m}}\right)\right]$. The grain filling stage was the one most sensitive to the water deficit when this was applied in just one stage, indicating low sensibility of the crop to the water stress.
\end{abstract}

Key words: Vigna ungüiculata, water deficit, evapotranspiration

\footnotetext{
${ }^{1}$ Parte da dissertação de mestrado do primeiro autor

${ }^{2}$ Eng. Agrônomo, M.S. em Agronomia, CP 12168, CEP 60450-760, Fortaleza, CE

${ }^{3}$ Prof. Doutor, Departamento de Engenharia Agrícola, DENA/UFC, CP 12168, CEP 60450-760, Fortaleza, CE
} 


\section{INTRODUÇÃO}

O feijão Caupi (Vigna unguiculata (L.) Walp.) é o alimento básico na alimentação das populações mais pobres, exercendo importante função social no suprimento das necessidades nutricionais dessa camada, além de desempenhar papel fundamental na composição da produção agrícola brasileira, particularmente do Nordeste.

A maioria das culturas possui períodos críticos quanto à deficiência hídrica, durante os quais a falta de água causa sérios decréscimos na produção final; os prejuízos causados dependem da sua duração e severidade e do estádio de desenvolvimento da planta (Folegatti et al., 1997).

Para se obter ótima produção deve-se conhecer a resposta do suprimento de água sobre o rendimento, a qual, segundo Doorenbos e Kassam (1994), é quantificada pelo fator de resposta da cultura $\left(\mathrm{k}_{\mathrm{y}}\right)$ que relaciona a queda de rendimento relativo $\left[1-\left(\mathrm{y}_{\mathrm{r}} / \mathrm{y}_{\mathrm{m}}\right)\right]$ com déficit de evapotranspiração relativa [1-(ET/ $\left.\left./ \mathrm{ET}_{\mathrm{m}}\right)\right]$.

A obtenção de uma alta produção também requer adoção de práticas de manejo que visem melhorar a irrigação e esta adoção só é possível com o conhecimento das necessidades hídricas das culturas.

Ante o exposto, esta pesquisa teve como objetivo estudar o fator de rendimento da cultura do feijão Caupi, cultivar Epace-11, quando submetida ao regime de déficit hídrico em diferentes estádios fenológicos.

\section{MATERIAL E MÉTODOS}

O experimento foi conduzido sob condições de campo, na área experimental do Laboratório de Hidráulica e Irrigação do Departamento de Engenharia Agrícola, Centro de Ciências Agrárias, Universidade Federal do Ceará, Fortaleza, CE, Brasil. A cidade de Fortaleza situa-se entre os paralelos $3^{\circ} 45^{\prime}$ e $4^{\circ} 00^{\prime} \mathrm{S}$ e os meridianos de $39^{\circ} 15^{\prime}$ e $39^{\circ} 30^{\prime}$ a Oeste de Greenwich. O clima da região, na classificação climática de Koppen, é do tipo Bsh, isto é, semi-árido, com chuvas irregulares. A temperatura máxima média mensal é de $27^{\circ} \mathrm{C}$ e a mínima de $25^{\circ} \mathrm{C}$, e sua precipitação média anual é de $1470 \mathrm{~mm}$, concentrada no período de fevereiro a maio.

A área na qual foi instalado o experimento apresenta solo de textura franco-argilo-arenosa, classificado como Podzólico-Vermelho-Amarelo equivalente eutrófico.

As curvas características de retenção de água no solo, nas profundidades de $10,30,50$ e $70 \mathrm{~cm}$, foram obtidas no Laboratório de Física do Solo do Departamento de Engenharia Agrícola da UFC, utilizando-se amostras indeformadas e acondicionadas em cilindros do tipo Uhland. As amostras foram submetidas às tensões $0 ; 2 ; 4 ; 6 ; 8 ; 28 ; 50 ; 100 ; 300$ e 700kPa em funil de Haines e em câmara de pressão de Richards. De posse dos valores de umidade, da base de volume $\left(\theta, \mathrm{cm}^{3} . \mathrm{cm}^{-3}\right)$ e do potencial mátrico de água no solo $\left(\Psi_{\mathrm{m}}, \mathrm{cm} \mathrm{c}\right.$.a) obtiveramse os parâmetros $\alpha$, n e m da Equação (1) através do programa computacional CURVARET, descrito por Dourado Neto et al. (1990).

$$
\theta_{a}=\theta_{\text {res. }}+\frac{\left(\theta_{s}-\theta_{\text {res. }}\right)}{\left[1+(\alpha h)^{n}\right]^{m}}
$$

sendo:

$\theta_{\mathrm{a}} \quad$ - Umidade atual do solo $\left(\mathrm{cm}^{3} \cdot \mathrm{cm}^{-3}\right)$

$\theta_{s}^{a} \quad$ - Umidade no ponto de saturação do solo $\left(\mathrm{cm}^{3} \cdot \mathrm{cm}^{-3}\right)$

$\theta_{\text {res. }} \quad$ - Umidade residual do solo $\left(\mathrm{cm}^{3} . \mathrm{cm}^{-3}\right)$

$\alpha, n$ e m - parâmetros do solo da equação de Van Genuchten

$\mathrm{h} \quad$ - módulo do potencial mátrico da água do solo, ( $\mathrm{cm}$ de água)

O delineamento experimental adotado foi o de blocos ao acaso, com quatro repetições e oito tratamentos, perfazendo o total de 32 unidades experimentais. Os tratamentos foram definidos em função da época de indução do déficit hídrico nos diferentes estádios fenológicos da cultura (vegetativo, floração e enchimento de grãos) e assim agrupados: $\mathrm{T} 1=$ tratamento em que a cultura não sofreu nenhum déficit hídrico nas três fases fenológicas; portanto, a evapotranspiração real é a máxima; $\mathrm{T} 2$ = a cultura sofreu déficit na fase de enchimento de grãos; T3 = déficit no estádio de floração; T4 = déficit nos estádios de floração e enchimento de grãos; T5 = déficit na fase vegetativa; T6 = déficit nos estádios vegetativo e enchimento de grãos; $\mathrm{T} 7$ = déficit nos estádios vegetativo e floração e $\mathrm{T} 8=$ déficit nos 3 estádios fenológicos.

Inicialmente, toda a área experimental foi irrigada por aspersão para o estabelecimento e a uniformização da cultura nos estádios iniciais. Conforme os tratamentos previstos, após a uniformização do estande as irrigações passaram a ser aplicadas por um sistema em forma de bastão, feito de PVC rígido de 3/4" de diâmetro com $2,2 \mathrm{~m}$ de comprimento e furos de $2 \mathrm{em} 2 \mathrm{~cm}$ tendo, em uma de suas extremidades, um registro e um hidrômetro de vazão máxima igual a $1,5 \mathrm{~m}^{3} \cdot \mathrm{h}^{-1}$ e resolução de $0,0001 \mathrm{~m}^{3}$. Durante as irrigações, o equipamento era movimentado manualmente em toda a parcela para se ter melhor distribuição de água.

As parcelas não estressadas receberam irrigações quando o potencial mátrico do solo, medido no tensiômetro a $10 \mathrm{~cm}$, atingia $-15 \mathrm{kPa}$. A lâmina aplicada era calculada quando o solo atingia uma condição ótima, ou seja, correspondente àquela que elevasse a umidade do solo do $\theta_{\text {critico }}$ à capacidade de campo. As demais parcelas em déficit receberam metade da lâmina média aplicada no tratamento sem déficit.

As medições diárias das tensões da água no solo foram feitas por baterias de tensiômetros instalados nas profundidades de $10,30,50$ e $70 \mathrm{~cm}$. sendo que cada tratamento recebeu, em duas de suas quatro repetições, uma bateria de tensiômetros nas profundidades citadas, com exceção do tratamento que não sofreu déficit mas que recebeu, em cada repetição, uma bateria de tensiômetros. Os tensiômetros instalados a $10 \mathrm{~cm} \mathrm{de}$ profundidade serviram para indicar o momento da irrigação, juntamente com as leituras de $30 \mathrm{~cm}$, para o cálculo da lâmina de irrigação.

Cada unidade experimental foi constituída por 10 linhas de plantas de $2 \mathrm{~m}$ de comprimento espaçadas de $0,8 \mathrm{~m}$; o espaçamento entre as covas foi de $0,4 \mathrm{~m}$. As plantas situadas nas laterais das parcelas serviram como bordadura. Cada parcela ocupou uma área de $16 \mathrm{~m}^{2}$, sendo que a área útil continha 48 plantas e ocupava $7,68 \mathrm{~m}^{2}$.

Foram semeadas cinco sementes por cova, deixando-se, após o desbaste, duas plantas, totalizando cem plantas em cada unidade experimental. O replantio foi feito sete dias após o plantio.

Utilizou-se uma adubação de fundação em todas as unidades experimentais na proporção de $20 \mathrm{~kg} \cdot \mathrm{ha}^{-1} \mathrm{de} \mathrm{N}, 80 \mathrm{~kg} \cdot \mathrm{ha}^{-1} \mathrm{de}_{2} \mathrm{O}_{5}$, 
10kg.ha-1 de $\mathrm{K}_{2} \mathrm{O}$ e 15 a 20 toneladas de esterco curtido de curral por hectare, com base em análise de solo realizada pelo Laboratório do Departamento de Ciências do Solo do Centro de Ciências Agrárias da Universidade Federal do Ceará. Os produtos utilizados foram uréia, superfosfato triplo e cloreto de potássio.

Os tratos culturais, como capinas e controle de pragas e doenças, foram realizados no decorrer do ciclo da cultura, sempre que necessário. As capinas foram efetuadas manualmente com enxada, de acordo com a ocorrência de ervas daninhas. Para as aplicações dos defensivos agrícolas utilizou-se um pulverizador costal, sendo que, para o controle de pragas e doenças, foram usados os defensivos: Monocrotophos, na dosagem de $1 \mathrm{ml}$ do produto para 1 litro de água, e Mancozeb, na dose de 3,0g do produto para 1 litro de água.

A colheita das unidades experimentais foi realizada quando as mesmas apresentavam mais de $50 \%$ de suas vagens maduras, sendo executada de maneira manual.

\section{Estimativa da evapotranspiração}

A evapotranspiração da cultura foi estimada mediante o método do balanço hídrico que tem seu fundamento básico na lei da conservação das massas, apresentada por Reichardt (1985) conforme equação:

$$
\int_{\mathrm{t}_{0}}^{\mathrm{t}_{1}}\left(\mathrm{p}+\mathrm{i}-\mathrm{e} \pm \mathrm{q}_{\mathrm{e}} \pm \mathrm{q}_{\mathrm{z}}\right) \mathrm{dt}=\int_{0}^{\mathrm{z}} \int_{\mathrm{t}_{0}}^{\mathrm{t}_{1}}\left(\frac{\partial \theta}{\partial \mathrm{t}}\right) \mathrm{dtdz}
$$

O primeiro termo da Equação 2 representa o somatório de todas as entradas e saídas de água num volume de solo de base unitária e profundidade, durante um intervalo de tempo $\Delta \mathrm{t}$. O segundo termo representa as variações da quantidade de água, no mesmo intervalo de tempo $(\Delta \mathrm{t})$ em uma camada de profundidade 0 a z. Esta equação, simplificada, integrada com relação ao tempo e à profundidade, resultando:

$$
\mathrm{P}+\mathrm{I}-\mathrm{ET} \pm \mathrm{Q}_{\mathrm{Z}} \pm \mathrm{ES}= \pm \Delta \mathrm{A}
$$

em que:

ET - evapotranspiração da cultura (mm)

P - precipitação natural (mm)

I - irrigação (mm)

Qz - drenagem profunda ou ascensão capilar (mm)

ES - escoamento superficial (mm)

$\Delta \mathrm{A}$ - variação do armazenamento $(\mathrm{mm})$ da água do solo na camada de 0 - z para o intervalo de tempo considerado no balanço.

A Equação 3 é comumente utilizada para efeito de balanço hídrico; entretanto, para seu uso em solos cultivados é necessário considerar-se uma profundidade do volume de solo que contenha todo o sistema radicular da cultura e que, na pesquisa, tenha sido considerado igual a $0,60 \mathrm{~m}$.

\section{Determinação dos componentes do balanço}

Os dados de precipitação pluviométrica utilizados neste estudo foram obtidos junto à Estação Agrometeorológica do Centro de Ciências Agrárias da Universidade Federal Ceará, situada a aproximadamente $100 \mathrm{~m}$ do local do experimento.

As lâminas de irrigação foram definidas em função do potencial da água no solo, nas profundidades de 10 e $30 \mathrm{~cm}$. A profundidade efetiva do sistema radicular considerada para a cultura foi de $60 \mathrm{~cm}$ e as expressões para o cálculo das lâminas de irrigação foram:

Camada 0-20cm

$$
\mathrm{L}_{(0-20)}=\left(\theta_{\mathrm{cc10}}-\theta_{\mathrm{a} 10}\right) \cdot 200
$$

Camada $20-40 \mathrm{~cm}$

$$
\mathrm{L}_{(20-40)}=\left(\theta_{\mathrm{cc} 30}-\theta_{\mathrm{a} 30}\right) \cdot 200
$$

em que:

$\mathrm{L}_{(0-20)} \mathrm{e} \quad$ - lâmina de irrigação para as camadas $0-20$ e $20-40 \mathrm{~cm}$

$\mathrm{L}_{(20-40)}$ respectivamente $(\mathrm{mm})$

$\theta_{\text {cc10 }}$ e $\theta_{\text {cc30 }}$ - umidades do solo $\left(\mathrm{cm}^{3} . \mathrm{cm}^{-3}\right)$ correspondentes às capacidades de campo nas profundidades de $10 \mathrm{e}$ $30 \mathrm{~cm}$, respectivamente. Esses parâmetros foram determinados na área experimental onde se estimou a condutividade hidráulica do solo.

$\theta_{\text {a10 }}$ e $\theta_{\text {a30 }}$ - umidade do solo $\left(\mathrm{cm}^{3} . \mathrm{cm}^{-3}\right)$ no momento das irrigações, nas profundidades de 10 e $30 \mathrm{~cm}$, respectivamente.

A drenagem profunda e a ascensão capilar da água do solo foram calculadas para profundidade de $0,60 \mathrm{~m}$, pela equação de Buckingham-Darcy, escrita de forma simplificada por Reichardt (1985) como:

$$
Q_{Z}=-K(\theta) \frac{\Delta \psi_{T}}{\Delta Z}
$$

em que:

$K(\theta)$ - condutividade hidráulica em função do valor da umidade do solo

$\Delta \psi / \Delta \mathrm{Z}$ - gradiente do potencial total da água no solo na profundidade $\mathrm{z}$.

Escrevendo-se a Equação 6 para a profundidade de $z=60 \mathrm{~cm}$, tem-se:

em que:

$$
Q_{Z}=-K(\theta)_{60}\left\{\frac{\psi_{t}^{50}-\psi_{t}^{70}}{20}\right\}_{60}
$$

$\mathrm{K}(\theta)_{60}$

-condutividade hidráulica do solo em função da umidade $\theta$ na profundidade $0,60 \mathrm{~m}$

$\left\{\frac{\psi_{t}^{50}-\psi_{t}^{70}}{20}\right\}_{60}$

- gradiente de potencial total da água do solo na profundidade de $0,60 \mathrm{~m}$, sendo que $\psi_{t}^{50}$ e $\psi_{t}^{70}$ representam o potencial total da água do solo a $50 \mathrm{~cm}$ e $70 \mathrm{~cm}$ (cm de água), respectivamente.

Os valores de $\mathrm{K}(\theta)_{60}$ foram obtidos mediante um ensaio de campo realizado na área do experimento, numa parcela de $25 \mathrm{~m}^{2}$, com três baterias de tensiômetros de coluna de mercúrio, nas profundidades de 0,$10 ; 0,30 ; 0,50 ; 0,70$ e $0,90 \mathrm{~m}$. O ensaio foi conduzido por um período de 40 dias, onde foi obtida a seguinte equação para a condutividade hidráulica a $60 \mathrm{~cm}$, conforme Saunders (1978):

em que:

$$
\mathrm{K}(\theta)_{60}=4 \mathrm{E}-07 \mathrm{e}^{68,936 \theta}
$$

$\mathrm{K}(\theta)_{60}$ - condutividade hidráulica em função da umidade média na profundidade de $0,60 \mathrm{~m}\left(\mathrm{~mm} \cdot \mathrm{dia}^{-1}\right)$

$\theta \quad$ - umidade média na profundidade de $0,60 \mathrm{~m}$.

A determinação da variação do armazenamento da água do solo na profundidade e no intervalo de tempo considerados, foi obtida mediante a expressão descrita por Reichardt (1985) : 


$$
\Delta \mathrm{A}=\left(\theta_{2}-\theta_{1}\right) \mathrm{z}
$$

em que:

$\theta_{2}$ - umidade média do perfil até a profundidade de $0,60 \mathrm{~m}$, no dia da irrigação $\left(\mathrm{cm}^{3} \cdot \mathrm{cm}^{-3}\right)$

$\theta_{1}$ - umidade média do perfil até a profundidade de $0,60 \mathrm{~m}$, no dia da irrigação anterior $\left(\mathrm{cm}^{3} . \mathrm{cm}^{-3}\right)$

$\mathrm{z}$ - profundidade adotada para o balanço $(\mathrm{z}=60 \mathrm{~cm})$.

Na condução do presente trabalho, o escoamento superficial (ES) foi desprezado, uma vez que as unidades experimentais foram sistematizadas a nível e feito camaleões para evitar o escoamento superficial da água de irrigação.

A componente ET é a incógnita da equação do balanço hídrico. Explicitando-a na Equação 3, tem-se:

$$
\mathrm{ET}=\mathrm{P}+\mathrm{I} \pm \mathrm{Q}_{\mathrm{Z}}-\Delta \mathrm{A}
$$

Para as condições em que o experimento foi desenvolvido, a evapotranspiração pode ser conceituada como evapotranspiração máxima $\left(\mathrm{ET}_{\mathrm{m}}\right)$ e real $\left(\mathrm{ET}_{\mathrm{r}}\right)$.

As evapotranspirações máxima e real do feijão foram determinadas através da equação do balanço hídrico (Equação 10) sendo que, para o cálculo da evapotranspiração máxima, utilizaram-se as 4 repetições, pois todas as parcelas continham tensiômetros, enquanto para o cálculo da evapotranspiração real apenas duas receberam tensiômetros.

\section{Fator de sensibilidade da cultura ao déficit hídrico $(\mathrm{Ky})$}

Para a determinação do fator de sensibilidade da cultura ao déficit hídrico $\left(\mathrm{K}_{\mathrm{v}}\right)$ foi utilizada a expressão empírica descrita por Doorenbos e Kassam (1994) que quantifica a relação entre a redução do rendimento relativo e o déficit de evapotranspiração.

$$
\mathrm{K}_{\mathrm{y}}=\left[1-\left(\mathrm{y}_{\mathrm{r}} / \mathrm{y}_{\mathrm{m}}\right)\right] /\left[1-\left(\mathrm{ET}_{\mathrm{r}} / \mathrm{ET}_{\mathrm{m}}\right)\right]
$$

sendo:

$\mathrm{y}_{\mathrm{r}}$ - rendimento real da cultura obtido nos tratamentos submetidos ao estresse hídrico;

$\mathrm{y}_{\mathrm{m}}$ - rendimento máximo da cultura obtido no tratamento 1, que não sofreu estresse hídrico;

$E T_{r}$ - evapotranspiração real da cultura obtida nos tratamentos submetidos a estresse hídrico;

$\mathrm{ET}_{\mathrm{m}}$ - evapotranspiração máxima da cultura obtida no tratamento 1 ; $\mathrm{k}_{\mathrm{y}}$ - fator de sensibilidade parcial para cada estádio ou fase fenológica.

\section{RESULTADOS E DISCUSSÃO}

Analisando os valores médios de $\mathrm{k}_{\mathrm{y}}$ dos tratamentos 2,3 e 5 (Tabela 1) que foram submetidos a déficit hídrico em apenas um estádio fenológico, observa-se que a redução relativa do rendimento feijão foi mais acentuada no estádio de enchimento de grãos (Tratamento 2) com $\mathrm{k}_{\mathrm{y}}=0,68$. O déficit hídrico nos tratamentos 3 e 5, estádios de floração e vegetativo, respectivamente, teve pouco efeito sobre o rendimento da cultura, apresentando valores praticamente iguais, $\operatorname{com~}_{\mathrm{y}}=0,34$ na fase de floração e $k_{y}=0,35$ na fase vegetativa. Tais valores, quando comparados com os citados por Doorenbos e Kassam (1994) apresentaram diferenças apreciáveis, uma vez que aqueles pesquisadores as obtiveram na floração $\mathrm{k}_{\mathrm{y}}=1,1$ enquanto se obtém $\mathrm{k}_{\mathrm{y}}=0,34$ no presente estudo. Os outros dois estádios apresentaram discrepâncias menores: enchimento de grãos $\left(\mathrm{k}_{\mathrm{y}}\right.$ $=0,75$ para Doorenbos e Kassam, contra $\left.\mathrm{k}_{\mathrm{y}}=0,68\right)$ e vegetativo $\left(\mathrm{k}_{\mathrm{y}}=0,2\right.$ para Doorenbos e Kassam, contra $\mathrm{k}_{\mathrm{y}}=0,35$ para o presente estudo). Deve-se ressaltar que os valores citados de ky, por Doorenbos e Kassam (1994) são para o feijão Phaseolus, enquanto que a pesquisa realizada foi com feijão caupi.

A diferença de $\mathrm{k}_{\mathrm{y}}$ encontrada para a floração em relação ao valor dado pela FAO, pode ter sido influenciada pelas condições ambientais, pela adaptação da cultura a esse ambiente e, também, porque, segundo Doorenbos e Kassam (1994) uma avaliação dos dados experimentais de campo indica certa dispersão nos valores de $\mathrm{k}_{\mathrm{y}}$, que se deve à imperfeição experimental e às variações de clima, nível de evapotranspiração e solo. Além do mais, deve-se ressaltar, conforme os próprios autores citados, que não se dispõe de valores como padrão de referência para comparação; supõe-se, apenas, que a confiabilidade dos valores de $\mathrm{k}_{\mathrm{y}}$ apresentados pela $\mathrm{FAO}$, é semelhante à procedente da análise dos resultados experimentais de campo.

Os valores dos coeficientes de sensibilidade $\mathrm{k}_{\mathrm{y}}$ nos tratamentos com estresse hídrico em mais de um estádio fenológico, apresentaram o tratamento 8 com o maior valor de $\mathrm{k}_{\mathrm{y}}$, o qual foi submetido a deficiência hídrica durante todo o ciclo da cultura. Nos tratamentos com estresse hídrico em duas fases, o maior valor de $\mathrm{k}_{\mathrm{y}}$ ocorreu no tratamento 7 , com

Tabela 1. Valores médios de $\left[1-\left(\mathrm{ET}_{\mathrm{r}} / \mathrm{ET}_{\mathrm{m}}\right)\right]$ e $\left[1-\left(\mathrm{y}_{\mathrm{r}} / \mathrm{y}_{\mathrm{m}}\right)\right]$ e do fator de sensibilidade ky do feijão caupi, cultivar Epace-11, nos tratamentos sob déficit hídrico

\begin{tabular}{cccccccccc}
\hline Trat. & $\mathrm{ET}_{\mathrm{r}}$ & $\mathrm{ET}_{\mathrm{m}}$ & $\mathrm{ET}_{\mathrm{r}} / \mathrm{ET}_{\mathrm{m}}$ & $\left(1-\mathrm{ET}_{\mathrm{r}} / \mathrm{ET}_{\mathrm{m}}\right)$ & $\mathrm{y}_{\mathrm{r}}$ & $\mathrm{y}_{\mathrm{m}}$ & $\mathrm{y}_{\mathrm{r}} / \mathrm{y}_{\mathrm{m}}$ & $\left(1-\mathrm{y}_{\mathrm{r}} / \mathrm{y}_{\mathrm{m}}\right)$ & $\mathrm{k}_{\mathrm{y}}$ \\
\hline $\mathrm{T} 2$ & 56,13 & 91,16 & 0,616 & 0,384 & 697,5 & 944,25 & 0,739 & 0,261 & 0,68 \\
$\mathrm{~T} 3$ & 22,42 & 56,25 & 0,399 & 0,601 & 750,00 & 944,25 & 0,794 & 0,206 & 0,34 \\
T4 & 60,13 & 147,41 & 0,408 & 0,592 & 650,00 & 944,25 & 0,688 & 0,312 & 0,53 \\
T5 & 44,63 & 98,21 & 0,454 & 0,546 & 763,00 & 944,25 & 0,808 & 0,192 & 0,35 \\
T6 & 64,76 & 189,37 & 0,342 & 0,658 & 579,75 & 944,25 & 0,614 & 0,386 & 0,59 \\
T7 & 64,95 & 154,46 & 0,420 & 0,580 & 584,25 & 944,25 & 0,619 & 0,381 & 0,66 \\
T8 & 89,92 & 245,62 & 0,366 & 0,634 & 400,75 & 944,25 & 0,424 & 0,576 & 0,91 \\
\hline
\end{tabular}

ET, $\mathrm{ET}_{\mathrm{m}}$ : Evapotranspiração real e máxima, em mm.dia ${ }^{-1}$, respectivamente

$\mathrm{y}_{\mathrm{r}}, \mathrm{y}_{\mathrm{m}}$ : Produção real e máxima, em kg.ha-1 ${ }^{-1}$, respectivamente 
deficiência de água nos estádios vegetativo e floração; o valor de $\mathrm{k}_{\mathrm{y}}=0,66$ no tratamento 7 é aproximadamente igual à soma entre os tratamentos 3 e 5, que sofreram déficit hídrico nos estádios de floração e vegetativo, respectivamente; entretanto, a interação nos tratamentos 4,6 e 8 não correspondeu à soma dos valores de $\mathrm{k}_{\mathrm{y}}$ obtidos nos tratamentos em que o déficit foi aplicado em cada fase, isoladamente. No geral, os valores dos coeficientes de sensibilidade $\mathrm{k}_{\mathrm{y}}$ encontrados para os tratamentos aplicados foram inferiores a 1 , mostrando baixa sensibilidade da cultura ao estresse hídrico.

\section{CONCLUSÕES}

1. Os valores de ky obtidos nos tratamentos estudados foram todos inferiores a 1 , indicando baixa sensibilidade aos déficits hídricos aplicados na pesquisa.

2. A sensibilidade ao déficit hídrico $\left(\mathrm{k}_{\mathrm{y}}\right)$ em cada estádio variou na seguinte ordem decrescente: enchimento de grãos, vegetativo e floração, para déficit aplicado em apenas um estádio fenológico. Para as condições de déficit em dois estádios, o maior valor encontrado foi no estádio vegetativo e enchimento de grãos.

\section{REFERÊNCIAS BIBLIOGRÁFICAS}

DOORENBOS, J.; KASSAM, A.H. Efeito da água no rendimento das culturas. Campina Grande: UFPB, 1994. 306p. (Estudos FAO: Irrigação e Drenagem, 33).

DOURADO NETO, D.; LIER, Q.J.V.; BOTREL, T.A.; LIBARDI, P.L. Curva de retenção de água do solo: algoritmo em quickbasic para estimativa dos parâmetros empíricos do modelo de Genuchten. Piracicaba: ESALQ, 1990. 32p. (Manual de instruções ao usuário).

FOLEGATTI, M.V.; PAZ, V.P.S.; PEREIRA, A.S.; LIBARDI, V.C.M. Efeito de diferentes níveis de irrigação e de déficit hídrico na produção do feijoeiro (Plaseolus vulgaris L). In: CONGRESSO CHILENO DE ENGENIERIA AGRÍCOLA, 2., 1997, Chillán. Disquete. Chillán, 1997.

REICHARDT, K. Processo de transferência no sistema soloágua-atmosfera. 4.ed. Campinas: Fundação Gargill, 1985. $466 \mathrm{p}$.

SAUNDERS, L.C.U. Métodos de determinação e variabilidade espacial da condutividade hidráulica sob condições de campo. Piracicaba: ESALQ. 1978. 71p. Tese Doutorado. 